ÁMBITOS. № 15 - Año 2006 (pp. 33-44)

\title{
Luces y sombras de las nuevas tecnologías de la información
}

\author{
Francisco Esteve Ramírez \\ (Universidad Complutense de Madrid) \\ María Teresa Sandoval Martín \\ (Universidad Carlos III de Madrid)
}

\section{Resumen}

La información se ha convertido en el eje central de las nuevas dinámicas globalizadoras de la industria cultural que utiliza a la información como un nuevo valor de uso. El colonialismo informativo está afectando al derecho que tiene todo ciudadano a recibir una información veraz, llegándose a la paradoja de que en la actual sociedad de la información, en la que existen mayores posibilidades tecnológicas y profesionales para recibir un mejor servicio informativo, se ofrece un producto más limitado y condicionado a los intereses políticos, económicos o ideológicos.

Entre las distintas opciones tecnológicas que consideramos pueden propiciar una alternativa para una mayor democratización de los medios de comunicación y una mejor posibilidad para ofrecer una plataforma mediática a las voces marginadas; destacamos en este ensayo, especialmente, a los webglobs, las comunidades virtuales y los portales personalizados.

\begin{abstract}
The information has become the central core of the new global dynamics in the cultural industry that gives the information new value.

Taking over the information affects the right that every citizen has to receive truthful information, separating themselves from the paradox of the Information Society in which exists better technological and professional possibilities in order to receive more informative services that offer a limited product depending on political, economical or ideological interests.

Between the different technological options that we consider we can propose an alternative for a more free communication "media"- democratization of the communication "media"- and therefore a better possibility to offer a "media" platform to those without a "voice" in society; we especially focus in this paper on weblogs, virtual communities and personal access.
\end{abstract}

Palabras clave: Tecnologías de la información, democratización de los medios de comunicación.

Key words: Information Technologies, democratization of the media. 


\section{LA ESPIRAL DEL SILENCIO}

a hegemonía del pensamiento único está imponiendo una fuerte losa sobre

el surgimiento y desarrollo de las voces discordantes que brotan de las distintas corrientes alternativas y críticas a las que se les impone una espiral del silencio que imposibilita la audición de otras voces que no sean las propias del stablismenth. El tradicional desequilibrio económico entre Norte y Sur se ha ampliado a un nuevo desequilibrio entre controladores de la información y usuarios de la misma. Se ha denunciado ya en múltiples ocasiones este injusto foso que separa el mundo de los poderosos respecto a los más empobrecidos, no solo económicamente sino también social, cultural e informativamente.

En el análisis efectuado por el profesor Quirós $(1991,94)$ sobre las características de los flujos mundiales de la comunicación puntualiza los principales factores del actual desequilibrio informativo internacional:

1. Una circulación en un solo sentido, siempre vertical, desde el centro a la periferia.

2. Un intercambio directo y recíproco entre los centros, aunque el centro del sistema también se encuentre jerarquizado.

3. Un intercambio periférico indirecto a través de los centros metropolitanos respectivos. Si la periferia forma parte de subsistemas diferentes, el intercambio de información es todavía más indirecto.

4. El intercambio directo y recíproco entre las periferias es mínimo.

A conclusiones similares llega el Informe de Masmoudi a la UNESCO cuando denuncia que "el sistema informativo actual encierra una forma de colonialismo político, económico y cultural que se refleja en la, a menudo, tendenciosa interpretación de las noticias concernientes a los países en desarrollo. Esta consiste en sobrevalorar acontecimientos, cuya importancia, en ciertos casos, es limitada e incluso inexistente; en recopilar hechos aislados y presentarlos como un conjunto; en establecer los hechos de tal forma que la conclusión que se puede sacar de ellos es necesariamente favorable para los intereses del sistema transnacional; en amplificar acontecimientos de escasa envergadura hasta despertar temores justificados; en guardar silencio en situaciones desfavorables para los intereses de los países de donde son originales estos medios".

Sin embargo, la información se ha convertido en el eje central de las nuevas dinámicas globalizadoras de la industria cultural que utiliza la información como un nuevo valor de uso. La economía y la información se han unido de forma inseparable en un binomio de intereses comunes. El colonialismo informativo está afectando al derecho que tiene todo ciudadano a recibir una información veraz, llegándose a la paradoja de que en la actual sociedad de la información, en la que existen mayores posibilidades tecnológicas y profesionales para recibir un mejor servicio informativo, se ofrece un producto más limitado y condicionado a los intereses políticos, económicos o ideológicos. 


\section{LAS TECNOLOGÍAS NO SON NEUTRAS}

Frente a la tendencia globalizadora de la cultura de masas conviene rechazar todo intento de presentar la tecnología como una herramienta neutral cuyos efectos dependieran exclusivamente del uso que de la misma se haga. Por el contrario, la tecnología es funcional al sistema socioeconómico en cuyo seno se desarrolla. El problema fundamental no estriba en el soporte de la información sino en el modelo de sociedad en que dicha información circula y al servicio de quien se pone.

Nos encontramos en los albores de una nueva era que algunos identifican con la denominada "sociedad de la información" en la que la nueva infraestructura de la información y la cultura debe estar al servicio del desarrollo integral de los individuos. Hay que contemplar, por tanto, la incidencia de las nuevas tecnologías en el campo de la información, el creciente desarrollo de los medios de comunicación y el transporte, la crisis económica, etc.

El reconocimiento de los posibles peligros que pueden derivarse de una incorrecta utilización de los nuevos medios tecnológicos no debe impedir, sin embargo, el aprovechamiento de sus múltiples posibilidades, tal como señala el profesor Martín Serrano $(1981,193)$ :

\footnotetext{
"sin necesidad de platearse utópicas sociedades de ciencia-ficción en las cuales las nuevas tecnologías comunicativas ofrecen la posibilidad a los receptores de recibir contenidos comunicativos generados, sincrónicos con el acontecer, parece evidente que ya es posible avanzar en ciertos campos hacia una comunicación cuyos contenidos estén menos mediados por la interpretación de los emisores, al menos en aquellas comunicaciones que tratan de un objeto de referencia accesible al medio de comunicación".
}

Las nuevas tecnologías de la información han propiciado el desarrollo de distintas alternativas a las formas tradicionales de distribución de los mensajes informativos. Así, estamos asistiendo a un progresivo avance de nuevas aplicaciones, especialmente en el campo digital, que suponen una radical transformación de los sistemas informativos, tanto en la elaboración de los productos informativos como en la canalización de los mismos. De igual manera, se está originando una notable modificación de las formas de acceso a los datos informativos facilitando un acceso más libre y amplio sin limitaciones espaciales y temporales. Esta "revolución mediática" se hace posible gracias al encuentro de determinados factores, tal como señala David Parra (1992, 283), "las tecnologías de la información ofrecerán una gama cada vez más variada de posibilidades gracias a la convergencia de las innovaciones tecnológicas en electrónica, informática y telecomunicaciones".

Entre las distintas opciones tecnológicas que consideramos pueden propiciar una alternativa para una mayor democratización de los medios de comunicación y una mejor posibilidad para ofrecer una plataforma mediática a las voces marginadas, destacamos en este ensayo, especialmente, a los webglogs, las comunidades virtuales y los portales personalizados. 


\section{LOS WEBLOGS COMO MEDIO ALTERNATIVO}

Los weblogs, que indistintamente llamaremos bitácoras (como también se les conoce en un intento por describir su significado en el mundo hispanohablante), son básicamente un tipo de páginas web personales que aparecieron en Estados Unidos a mediados de la década de los noventa, cuando se hizo público el acceso a Internet. Su uso se extendió cuando aparecieron herramientas y software que facilitaron su creación y mantenimiento ${ }^{1}$. Gracias a su simplificada gestión de contenidos ya se han convertido en un fenómeno de masas -se crean alrededor de 2000 weblogs diarios- al otro lado del océano y se empieza a expandir rápidamente a otros entornos, países y continentes. En la actualidad existen más de cuatro millones de bitácoras en la red $^{2}$. A la cabeza del desarrollo de esta nueva posibilidad de información digital se encuentran los Estados Unidos, aunque cada día aumenta el número de blogs en España ${ }^{3}$.

A pesar de su expansión, no existe una definición consensuada que identifique qué son las bitácoras. No obstante, en el intento de simplificar su definición, la mayoría de los expertos se refieren al weblog o bitácora como un "sitio web donde se recopilan cronológicamente mensajes de uno o varios autores, sobre una temática o a modo de diario personal". A ello hay que añadir una serie de elementos comunes que se dan en la mayoría de estos sistemas o interfaces como una lista de enlaces a otros weblogs, un archivo a modo de hemeroteca, enlaces permanentes para citar una entrada o una función para añadir comentarios de los lectores. La escritura de estos textos y su publicación es instantánea, la actualización del contenido suele ser -o al menos debería ser- (casi) diario y los últimos mensajes se presentan en la parte superior de la pantalla (mientras que los textos anteriores se almacenan en los archivos). La mayoría incluye un gran número de vínculos hacia otras fuentes de información provenientes de la propia Red o de otros weblogs mediante el uso extensivo del hipertexto.

Dado que se trata de una página web personal, es en consecuencia, subjetiva. Los contenidos no están sometidos a ningún orden de composición pues responde a la visión personal de sus autores y, por tanto, la edición del contenido, así como la selección de los vínculos, depende de la personalidad del creador.

Descrito así, podríamos pensar que se trata sólo de una lista de distribución o de un foro de debate, cuyos objetivos se parecen. De hecho, existen también weblogs comunitarios. Pero, un weblog esta lejos de asemejarse a alguna de esas

\footnotetext{
1 Entre las empresas más importantes que prestan servicios de alojamiento y creación de blogs para cualquier internauta se encuentran Blogger (propiedad de Google), Movible Type, Pytas, Dypop o Antville.

${ }^{2}$ Véase el estudio realizado por la empresa Perseus Development Corp titulado The Blogging Iceberg [http://www.perseus.com/blogsurvey/thebloggingiceberg.html] (visitado el 30 de marzo de 2004).

${ }^{3}$ Véase el trabajo de Fernando Tricas, Víctor Ruiz y Juan J. Merelo "Do we live in a small world? Measuring the Spanish-speaking Blogosphere" publicado en el sitioweb Blogalia [http:// www.blogalia.com/pdf/2003050blogtalk.pdf] (visitado el 14 de marzo de 2004).
} 
aplicaciones. Las temáticas específicas de las que pueden versar son muy diversas, tan heterogéneas son los intereses de sus autores: economía, tecnologías, medio-ambiente, política, salud, etcétera.

Estas páginas personales temáticas, fundamentalmente aquellas que producen un mayor volumen de información de calidad, han conseguido situarse como fuentes de la opinión pública, ofreciendo un punto de vista que en numerosas ocasiones difiere totalmente de lo publicado en los medios tradicionales, donde existen mayores limitaciones de expresión. De ahí, que esta frescura y "estilo libre" de los contenidos de las bitácoras haya suscitado desde hace algún tiempo cierta "rivalidad informativa" de los weblogs respecto de la producción informativa de los diarios digitales. El gran logro de este nuevo medio nacido en Internet es que ha permitido democratizar el acceso a la información, consiguiendo acceder a un sistema de comunicación en el que las personas pueden expresarse libremente sin ningún tipo de censura -aunque para algunos autores esto sería discutible en algunos casos-, lo que lo convierte en el medio adicional idóneo para obtener información y compartir opiniones.

El profesor José Luis Orihuela ${ }^{4}$ nos señala que los weblogs se han convertido en los nuevos medios de comunicación en los Estados Unidos y que pueden llegar a ser la 'next big thing' de la comunicación empresarial y política.

En el ámbito comercial ya se está haciendo un uso inteligente de este nuevo medio interactivo. Para empresas como Macromedia y Júpiter Research la gestión eficiente de la información se está convirtiendo en una prioridad estratégica (Orihuela, 2004). De hecho, varias empresas norteamericanas han visto de cerca cómo diversos weblogs aportaban información muy detallada sobre sus políticas internas, procesos, datos sobre decisiones de directivos, entre otros asuntos, en periodos de crisis con sus trabajadores (despidos masivos, abusos en las condiciones laborales contratas). Estas informaciones volcadas en las bitácoras cobran aún más valor al convertirse en fuente de información, o al menos en fuente de investigación, para muchos medios de comunicación, sindicatos y terceras partes involucradas en cualquier conflicto ${ }^{5}$.

En círculos políticos, también se están dando numerosos ejemplos de las posibilidades que brinda este nuevo medio nacido en la red. Durante las últimas elecciones presidenciales primarias de Estados Unidos se ha podido observar cómo todos los candidatos del Partido Demócrata estaban llevando a cabo una intensa campaña de comunicación a través de weblogs para difundir sus mensajes, recau-

\footnotetext{
4 El profesor José Luis Orihuela es uno de los máximos expertos e investigadores en esta materia y ha publicado numerosos trabajos sobre este asunto que pueden consultarse en el sitio web ecuaderno.

5 "El papel de los weblog-bitácoras en las estrategias de comunicación" Dosdoce. Revista de Comunicación, Arte y Literatura, Nr. 2, mayo-junio 2004. [http://www.dosdoce.com/ pagina_nueva_15.htm] (visitada el 1 de junio de 2004).
} 
dar fondos, movilizar voluntarios para sus campañas y hasta para intoxicar el mensaje del contrario ${ }^{6}$.

Para comprender mejor las características de esta nueva herramienta y poder visualizar su potencial como nuevo recurso informativo se hace necesario ahondar en una de sus peculiaridades más destacadas: el uso del hipertexto para vincular lo comentado a informaciones de primera mano, en muchas ocasiones elaboradas por otros bloggers, en otras, por medios digitales u otros sitios web. Es ésta una de las grandes fuerzas de los weblogs al facilitar la recuperación de informaciones que ya no son del todo actuales pero siguen siendo relevantes. "Los weblogs hacen las veces de filtro", indica Dan Gillmor. "En la masa de información disponible en la red, los webloggers tratan de encontrar los elementos más sabrosos y más interesantes. Abren el camino a los demás internautas" (Fournier, 2004).

Aunque es cierto que las informaciones adquiridas a través de esta vía no son siempre todos lo rigurosas que podrían ser (recordemos que en el mundo de los medios electrónicos la veracidad de la noticia siempre es menos importante que su rapidez y su contenido 'picante'), es cierto también que la corrección Online es mucho más sencilla que la que se realiza sobre el papel y, según Dan Gillmor, los creadores de weblogs "asumen con mayor facilidad la posibilidad de un error o una interpretación equivocada porque el medio así lo impone y están acostumbrados" (Fournier, 2004).

Otro de los aspectos que distinguen a estas páginas web de las convencionales es el enorme espíritu comunitario y la constante interacción que se produce entre los bloggers. La mayoría incorporan numerosos vínculos hacia páginas web de otros webloggers con los que comparten intereses y hallazgos en la red. Esta interconexión crea un poderoso efecto multiplicador: "Una comunidad de diez, cien o mil personas que intercambian ideas y comentarios pueden crear un dinamismo increíble", comenta Henry Copeland, director general de Pressflex, proveedor de soluciones completas para la prensa (Fournier, 2004). Este aspecto resulta especialmente interesante en el ejercicio periodístico porque estas comunidades se convierten en fuentes potenciales para el periodista que busca la opinión de determinados profesionales o expertos en alguna materia.

\section{DOBLE FEED-BACK ENTRE DIARIOS Y WEBLOGS}

Con el inicio de la guerra de Iraq, la opinión pública internacional tuvo por primera vez, durante un conflicto armado, la oportunidad de confrontar no sólo las versiones de los grandes medios y las de los contendientes, sino también las de fuentes independientes. Esto se debió en gran medida a que los enviados especiales y los corresponsales de guerra se sirvieron de los weblogs para contar su visión del

${ }^{6}$ Consúltese a este respecto los siguientes artículos: “¿Es Internet la nueva fórmula de financiación de los partidos?", Expansión, 8 de marzo de 2004, y Chris Ulbrich: "¿Los weblogs consiguen dinero para las campañas?, Wired News, 18 de febrero de 2004. [http://us.terra.wired.com/ wired/negocios/0,1154,25814,00.html ] (visitado el 30 de marzo de 2004). 
acontecer diario. A través de sus propios weblogs, los periodistas enviados a Iraq podían ir más allá en sus informaciones y aportar una visión más humana y directa de los hechos, al igual que había ocurrido ya antes durante el 11 de septiembre. Aquel día, mientras el Wall Street Journal estaba siendo desalojado por su proximidad a las Torres Gemelas, cientos de bloggers volcaban sus primeras impresiones de lo sucedido, multitud de fotografías y testimonios. Con su actuación contribuyeron a crear una nueva lógica de comunicación.

Posteriormente, en la guerra de Iraq los weblogs de los profesionales del periodismo demostraron su poder como herramienta para la transmisión de informaciones y experiencias personales desde un punto de vista independiente, alejado de los imperativos de las empresas mediáticas y de los intereses políticos. En algunos casos se utilizó porque de esta forma lograban esquivar las presiones de los medios tradicionales o la censura de uno y otro lado del conflicto. En otros, únicamente por el afán de contarle al mundo lo que allí estaba sucediendo en primera persona. Esta herramienta les proporcionaba el máximo de libertad para contar lo que allí estaban viendo y viviendo. De hecho, los contenidos de las informaciones que publicaban en sus respectivos medios eran diferentes de las que recogían en sus diarios personales publicados en Internet. Estos autores aportaron "más información, más opinión y más material gráfico, sin comparación con ningún otro acontecimiento anterior" (Cerezo y Zafra, 2003, 23).

A esta pluralidad informativa se sumaron los cuadernos de bitácora de los habitantes de Iraq que contaron cómo estaba sufriendo la población los avatares de la guerra. En este sentido, la bitácora del joven arquitecto bagdadí Salam se convirtió en una de las más populares durante la contienda. Por primera vez en la historia de las guerras, los periodistas y la población que sufría el horror pudieron narrar al resto del mundo de forma directa lo que allí estaba aconteciendo. Éste es uno de los ejemplos más significativos que ilustran cómo en un momento singular o extremo las bitácoras se convierten en fuente de información más próxima a los hechos, más incluso a veces que los medios tradicionales. Pero además, los weblogs, fundamentalmente aquellos muy especializados y con numerosos vínculos que completan la información pueden llegar a constituir una importante fuente de información complementaria para las redacciones tradicionales, que cada vez se van encontrando con más limitaciones en cuanto a tiempo y recursos. La mayoría de los medios digitales efectúan este trabajo de selección hoy día, pero lo hacen más bien de modo puntual, en algunas páginas o secciones concretas, o con motivo de ocasiones especiales (informes, eventos...).

Este fenómeno ha propiciado que la información, tanto desde el punto de vista del acceso, como su elaboración y tratamiento, esté cambiando. Esto se observa en que algunas empresas mediáticas han recapacitado y han comenzado a modificar la forma y el modo de narrar la actualidad informativa. Cada día son más los medios que admiten la necesidad de introducir cambios en sus políticas de comunicación, con iniciativas que van desde incluir una selección de algunos de los mejores blogs de información, de análisis, etc., pasando por enlazar desde las 
ediciones electrónicas a las bitácoras de sus corresponsales hasta llegar a encargarles a estos la elaboración de un warlog o diario de guerra. Dos de las empresas que más se han fijado en las potencialidades de comunicación de este nuevo género de información interactivo son el diario británico The Guardian [http:// www.guardian.co.uk/weblog/] y el argentino Clarín [http://weblogs.clarin.com/conexiones/]. Simón Walkman, director de las ediciones digitales del diario británico considera que la función de su medio no es sólo producir el contenido, sino también guiar a sus lectores hacia otras fuentes de información. Uno de los logros que consiguen los medios con la incorporación de este género del periodismo electrónico es la participación de sus lectores de una forma más interactiva ante el tratamiento de un tema en cuestión, logrando asimismo una mayor fidelización del usuario ante este medio.

Para algunos, la inclusión del blog dentro del diario implica pérdida de libertad para el periodista, pero, a la vez, aporta credibilidad a lo que narra. No hay que olvidar que también se crean blogs con fines propagandísticos, políticos y comerciales, aunque no se explicite en la página web. Estos "bloggers influyentes" diseminan su entusiasmo por una determinada postura ante un conflicto, ante las ideas que sostiene un partido político o ante un nuevo producto que acaba de lanzarse al mercado. No obstante, el resto de la comunidad de bloggers se encarga a menudo de desenmascarar los mensajes que conllevan intenciones ocultas o falsas verdades, conformando un sistema que se corrige y limpia a sí mismo.

\section{LAS COMUNIDADES EN INTERNET}

Una de las características de Internet es la de posibilitar la interrelación entre los receptores. En los medios de comunicación tradicionales esta posibilidad se limitaba, en la mayoría de los casos, a una interactividad relativa entre el emisor y el receptor que, en el caso de la prensa, se reducía a la sección de "Carta de los lectores", y en la radio y televisión, a las tertulias radiofónicas o a los concursos televisivos. Pero resultaba difícil establecer vínculos de unión entre los propios receptores. Para paliar tal situación se crearon determinadas asociaciones de usuarios de medios de comunicación que, fundamentalmente, se unían para defender sus derechos frente al poder casi absoluto de los medios.

Con el creciente desarrollo de los portales verticales en Internet se ha incrementado considerablemente esta intercomunicación entre los receptores. En las comunicaciones mercantiles, esta relación entre usuarios o clientes suele denominarse como $C$ to $C$ (customer to customer), frente a la interrelación entre empresas $B$ to $B$ (business to business). A través de los servicios de chat y foros de debate se establece una intercomunicación entre los internautas facilitando así el diálogo y el debate sobre temas de intereses afines.

En 1997, el término "Comunidad", se convirtió en una de las palabras de moda, tanto dentro como fuera de Internet. Una de las ventajas de la Red Internet es que permite la formación de comunidades salvando barreras geográficas. Sólo es 
necesario que sus miembros compartan intereses u objetivos para encontrarse unos a otros.

Según Esther Dyson ${ }^{7}$, las comunidades de internautas entablan conversaciones y otros tipos de interacción mediante una pagina web, una lista de correo o un foro de discusión: personas interrelacionadas mediante mensajes de texto y, cada vez más, mediante sitios virtuales multimedia a los que acceden de vez en cuando.

Un foro de discusión es como un tablón de anuncios virtual, en el que los miembros colocan sus mensajes o los leen cuando ellos quieren. Una lista de correo o lista de distribución es un foro de discusión activo, envía mensajes de forma regular a sus miembros pero, por norma general, también conserva archivos que permiten efectuar búsquedas. Una comunidad impone sus reglas ella misma. A menudo, éstas son invisibles hasta que se infringen o se ponen en entredicho, luego se discuten y se llega a una decisión. La resolución puede tomarla el moderador de la comunidad o adoptarse mediante una votación.

Uno de los principales foros de debate e intercambio de opiniones y experiencias entre los usuarios de Internet son las denominadas "comunidades virtuales". Se entiende por tales "los espacios de comunicación interactiva en tiempo real en los que la audiencia se convierte en emisora y receptora al mismo tiempo", según definición de David Cantolla ${ }^{8}$. En definitiva, el objetivo de estas "comunidades virtuales" es agrupar un determinado número de personas a las que les unen similares intereses. Estas comunidades tienen su origen en las comunidades científicas que, antes del nacimiento de Internet, intercambiaban investigaciones y experiencias a través de publicaciones especializadas y encuentros de especialistas del sector. Mediante la utilización de los servicios de Internet se facilita en gran manera este intercambio entre expertos o usuarios que tienen las mismas inquietudes o aficiones.

Las comunidades pueden ser lucrativas y no lucrativas. Las primeras tienen un marcado interés comercial (comunidad de subastas en la red, por ejemplo) cuyo objetivo es obtener beneficios economicos. La segunda, de carácter mas filantrópico, se mueve por el interes de compartir conocimiento. Así, existen comunidades de expertos desarrolladores del lenguaje Java, que intercambian información sobre la evolución de este programa.

La clave de las comunidades virtuales es la información y los servicios. La información que se ofrece en estos portales suele ser una información especializada ya que va dirigida a un sector de la población agrupada en torno a unos temas de interés común, bien de carácter científico, cultural, lúdico, etc. Asimismo, los usuarios encuentran en estos espacios cibernéticos una amplia oferta de servicios segmentados a las necesidades de la comunidad virtual. A través de estas

${ }^{7}$ Esther DYSON: Reportera de Forbes y graduada en Ciencias Económicas por la Universidad de Harvard, es una de las principales voces expertas en el mundo de Internet.

${ }^{8}$ CANTOLLA David: E.comm, mayo de 2000, pp. 106) 
comunidades se pretende unificar las comunicaciones a través de espacios informáticos donde poder compartir experiencias comunes, tanto sean de carácter científico como profesional o social.

Una de estas comunidades virtuales es la que ofrece el portal vertical www.pobladores.com Se trata de un espacio que agrupa a internautas interesados en establecer vínculos de debate y comunicación entre ellos. Según Jesús Carmo$\mathrm{na}^{9}$, uno de los creadores de esta comunidad, "Pobladores surgió con la idea de revolucionar el concepto de comunidad virtual, dándole a los usuarios la interactividad de la que carecen los actuales portales y comunidades virtuales". El portal está dividido en "Territorios" que corresponden a contenidos especializados como Deportes, Música, Tiempo Libre, Informática, Gente y Ciudades, etc.

Los medios tradicionales de comunicación pueden facilitar la creación de estos espacios virtuales mediante la creación de foros a partir de los artículos publicados en la prensa o los mensajes transmitidos a través de la radio o la televisión. Estos medios pueden facilitar la dirección de una de estas comunidades virtuales en Internet para establecer el contacto con los receptores y posibilitar el intercambio de opiniones entre los propios usuarios.

\section{DE LOS PORTALES VERTICALES A LOS PERTALES}

El futuro de Internet pasa por la personalización de los servicios en una nueva fase de especialización de sus prestaciones. Se trata de los portales personales, a los que podemos denominar pertales (personalized portales) "Es la fase-señala Arrakis Magazine ${ }^{10}$, de los llamados Agentes Inteligentes, pequeños programas residentes o una tecnología desarrollada a tal efecto que permitirá analizar al usuario acercándole a su hogar la información que éste previamente le haya especificado o constituya una rutina diaria. Imaginaos que leéis todas las mañanas la información internacional exclusivamente. Un agente preparado será capaz de, al poco tiempo, seleccionar todas las noticias internacionales que constituyan vuestro interés, descartando el resto".

Para la creación de portales temáticos a la medida han surgido distintas iniciativas en Internet. Una de las primeras es la página www.tuportal.com cuyo objetivo es generar portales verticales interactivos que sean punto de partida en la navegación por Internet. En la presentación del portal ofrecen convertir la página web del usuario en un portal temático que responda a las necesidades e intereses reales del cliente de forma personalizada e individualizada.

Esta personalización ha revolucionado el concepto de la información y la comunicación situando al usuario final como el principal elemento del proceso comunicativo. Tal como advierte Melania Pérez Iglesias", "Internet empieza a

9 CARMONA Jesús: Arrakis Magazine, octubre de 1999, pp. 30)

10 Arrakis Magazine, no 2, octubre de 1999, pp. 18)

11 PÉREZ IGLESIAS Melania, Nueva empresa.com, mayo de 2000, pp. 26. 
conocer a sus usuarios, gracias a los servicios que ofrece de personalización, suscripción, etc. Y cuando conoces a tus clientes, es mucho más fácil establecer una buena relación comercial con ellos. Y los Portales lo saben y también lo saben los proveedores de un volumen creciente de bienes y servicios, que ven con gran interés a un medio que es capaz de seleccionar a su audiencia con una precisión no imaginable en la prensa, en la televisión, o la radio".

Esta fragmentación de los contenidos de los portales en áreas de interés obligará a reorganizar la información de otra manera y abrirá las puertas a un tipo de navegación diferente, más cercana a la que en el sector de las tecnologías de próxima generación se denomina como navegación conceptual, según indica Luis Ángel Fernández Hermana ${ }^{12}$. Este nuevo tipo de navegación consiste en desarrollar una representación gráfica de la información guardada en los directorios, independientemente de su volumen, complejidad o heterogeneidad. La idea básica es encontrar la información no a través de las sucesivas capas que la estructuran (ya sea en árboles jerárquicos o a través de hiperenlaces), sino espacialmente.

Para la elaboración de todos estos contenidos especializados e, incluso, personalizados, se requiere el trabajo de los denominados "operadores de áreas de contenidos" que pueden equipararse a los redactores jefes de las distintas áreas de especialización de los medios tradicionales. Se trata de una nueva figura de periodista digital especializado que tiene como misión la selección y catalogación de los contenidos informativos por áreas de especialización.

Así pues, los portales verticales se están, configurando como un medio de comunicación interactiva y personalizada con una atención especial hacia las necesidades reales de los receptores. Así, los usuarios pueden solicitar una información permanente de acuerdo con el perfil de sus preferencias profesionales o personales. Según Axel Serena ${ }^{13}$, "el mercado tiende a la especialización. Ya no se diferentes servicios que aporten especialización. Por otro lado, los portales deberán ser capaces de generar ingresos gracias a la audiencia de que disponen. El hecho de servir más o menos páginas no será importante, sino el saber encontrar públicos diferenciados y el saber generar ingresos gracias a ellos".

Dada la imposibilidad de supervivencia del actual número de portales, tanto generalistas como especializados - algunos analistas vaticinan que sólo va a haber espacio para cinco grandes portales-, se está imponiendo la alternativa de acudir al mercado con una oferta diferenciada, pero no dirigida a todos los usuarios sino a segmentos diferenciados en los que existe una intención común. Se trata, según Javier Pérez ${ }^{14}$, "de las redes de valor alrededor de estas intenciones (Intention Value Networks), donde un conjunto de organizaciones o empresas se asocian para

12 FERNÁNDEZ HERMANA Luis Ángel, en Tanda.org, 26-1-1999.

13 SERENA Axel: El Mundo, Ariadna, 7-9-2000, pp. 11.

14 PÉREZ Javier: El Mundo, Nueva Economía, 7-5-2000. 
construir la citada red. Son como portales especializados pero bajo una idea más elaborada. El objetivo de la red de valor es satisfacer una necesidad asociada a cada intención".

\section{BIBLIOGRAFÍA}

CEREZO, José M. y ZAFRA, Juan M. (2003): El impacto de internet en la prensa (Cuadernos/Sociedad de la Información 3), Fundación Auna, Madrid, 2003.

FOURNIER, Vincent (2003): "Weblogs: otro útil de trabajo en el abanico de los medios electrónicos", Técnicas de Prensa, enero 2003, [http://www.ifra.com/WebSite/News.nsf/ 0/17C2D28676377B53C1256CB10042EE07].

LOSADA VÁZQUEZ, Ángel y ESTEVE RAMíREZ, Francisco (eds.)(2003): El periodismo de fuente, una ampliación del campo conceptual del ejercicio periodístico, Servicio de Publicaciones de la Universidad Pontificia de Salamanca.

MARTíN SERRANO, M. (edit.) (1981): Epistemología de la comunicación y análisis de la referencia. A.L. Madrid.

MASMOUDI: "The new world information order", en Journal of Communication. № 29, pg. 174). "El papel de los weblog-bitácoras en las estrategias de comunicación" Dosdoce. Revista de Comunicación, Arte y Literatura, Nr. 2, mayo-junio 2004. [http:// www.dosdoce.com/pagina_nueva_15.htm] (visitada el 1de junio de 2004).

PARRA, D. (1992): "Investigación, desarrollo y comunicación en mass-media”, en AA.VV.: Estudios sobre tecnologías de la información. Dicción. Madrid.

QUIRÓS, F. (1991): Curso de estructura de la información. Dossat. Madrid.

SANDOVAL MARTíN, María Teresa y SÁNCHEZ CALERO, María Luisa (2004): "Los diarios de guerra en línea: un medio emergente que cambia las formas de hacer Periodismo", comunicación presentada al Congreso de la Sociedad Española de Periodística, abril 2004 (en prensa).

(Recibido el 18-02-06, aceptado el 1-04-06) 